\title{
Synthesis and characterization of an electrochromic copolymer based on $2,2^{\prime}: 5^{\prime}, 2^{\prime \prime}$-terthiophene and 3,4-ethylenedioxythiophene
}

\author{
Mohammad Shamsuddin Ahmed • Haesang Jeong • \\ Jung-Min You • Seungwon Jeon
}

Received: 14 October 2011/ Accepted: 22 November 2011/Published online: 2 February 2012

(C) The Author(s) 2012. This article is published with open access at Springerlink.com

\begin{abstract}
A new electrochromic copolymer of $2,2^{\prime}: 5^{\prime}$, $2^{\prime \prime}$-terthiophene (TT) with 3,4-ethylenedioxythiophene (EDOT) was synthesized in $0.1 \mathrm{M}$ tetrabutylammonium perchlorate as supporting electrolyte and characterizations of the resulting copolymer P(TT-co-EDOT) performed by cyclic voltammetry, UV-vis spectroscopy, scanning electron microscopy, fourier transform infrared spectroscopy, nuclear magnetic resonance and thermal analysis. Spectroelectrochemical investigations showed that the copolymer film has electrochromic properties. It showed five different colors at various potentials (sky blue, gray, light purple, blues violet and dark blues violet). Double potential step chronoamperometry experiment illustrated that copolymer film had good stability, fast switching time and high optical contrast. At the neutral state of the copolymer $\lambda_{\max }$ was found at $500 \mathrm{~nm}$ and $E_{\mathrm{g}}$ was calculated as $1.63 \mathrm{eV}$. The copolymer film showed a maximum optical contrast of $54 \%$ at $1,100 \mathrm{~nm}$ with a short response time.
\end{abstract}

Keywords Electrochemical polymerization - Conducting polymer - Terthiophene - Ethylenedioxythiophene . Cyclic voltammetry $\cdot$ Electrochromism

\section{Introduction}

Both academia and industry are interested in conductive polymers for their fascinating electrochromic properties and their commercial applications (Skotheim and Reynoids 2007). Electrochromism is defined as the property inherent

M. S. Ahmed · H. Jeong · J.-M. You · S. Jeon $(\bowtie)$

Department of Chemistry and Institute of Basic Science, Chonnam National University, Gwangju 500-757, Korea e-mail: swjeon3380@naver.com in some materials which enables them to electrochemically switch between different color states as a result of redox reaction. Typical electrochromic behavior leads to switching between a transparent or lightened state and a color state or between two different color states. Many different types of organic and inorganic materials exhibit electrochromic behavior. Several discoveries have brought conducting polymers (CPs) to full commercialization with applications in electrochromic rearview mirrors (Monk et al. 2007), thin-film transistors (Halls et al. 1995), multicolored displays and image generation (Sahin et al. 2006), sensors (Albert et al. 2000), polymer light-emitting diodes (Lee et al. 2010), photovoltaics (Kim et al. 2010), and electrochromic devices (ECD) (Bar et al. 2009). Recently, research into CPs has mostly focused on their optical properties in the visible (Sonmez et al. 2003) and near infra-red (NIR) (Meng et al. 2003) spectral regions. Poly(thiophene) derivatives have been most widely studied materials, since they exhibit fast switching times, outstanding stability and high contrast ratio in the visible and NIR regions. Due to their increased versatility, organic materials such as viologens, metallophthalocyanines and CPs (Monk et al. 1995) have recently received much attention for potential electrochromic applications (Mortimer 1999). Among the various organic materials, conjugated polymers have several advantages over small molecules and inorganic solids, with their outstanding coloration efficiency, fast switching time (Yoo et al. 2010), multiple colorations with the same material, fine-tunability of the band gap (Roncali 1997), high stability (Ho et al. 1999), thermal stability even at high temperatures and glass transition temperature (Yen and Liou 2010), both $\mathrm{p}$ and $\mathrm{n}$ type dopable polymer (Udum et al. 2008), Solvent-resistant electrochromic polymer (Ramirez and Parise 2009), thinfilm flexibility and low cost. The electrochromism of CPs 
are related to the doping-undoping process. The doping process modifies the polymer's electronic structure, producing new electronic states in the band gap which cause color changes. A major focus in the study of electrochromic polymeric materials has been that of controlling their colors by main-chain and pendant group structural modification and copolymerization. Indeed, it has been observed that the color of copolymers based on carbazole, thiophene and pyrrole derivatives can be controlled by altering the ratio of the respective monomers (Yilmaz et al. 2004). Additionally, the polymer band gap can control the color contrast between the undoped and doped state (Yigitsoy et al. 2007). EDOT is a good substituted monomer since it produces a low band gap polymer with high stability and good conductivity (Wagner et al. 2005). EDOT has been chosen as an effective donor unit due to its strong electron donating effects and small steric interaction between repeating units in polymers (Beaujuge et al. 2008). Many electron acceptor units have been polymerized with EDOT to form low band gap copolymers. EDOT can give rise to noncovalent intramolecular interactions with adjacent thiophenic units and thus induce self-rigidification of the $\pi$-conjugated system in which it is incorporated (Raimundo et al. 2001). Several numbers of literatures have been published on EDOT-based copolymer research (Ahmed et al. 2011).

In the present study, we synthesized successfully an electrochromic copolymer of TT with EDOT. The electrochemical polymerization of $\mathrm{P}(\mathrm{TT}$-co-EDOT) was achieved in acetonitrile (MeCN) using tetrabutylammonium perchlorate (TBAP) as the supporting electrolyte. The resultant copolymer was characterized using CV, SEM, NMR, FTIR spectroscopy and colorimetry. The voltammograms and SEM micrographs were impressive. The optoelectrochemical and electrochromic properties of the copolymer such as its relative luminance, change of color upon redox switching and long-term switching stability were determined. The resulting multichromic polymer showed up to five different colors (sky blue, gray, light purple, bluish violet and dark bluish violet) and is therefore a promising polymer for organic electronic displays.

\section{Experimental}

Chemicals

EDOT, TT, MeCN and TBAP were purchased from Aldrich and used without further purification. All other reagents used were of analytical grade. High purity argon was used for deaeration. All experiments were carried out at room temperature. Every electrode (ITO, reference and counter) was washed with extra pure $\mathrm{MeOH}$ before used.
Instrumentation

$\mathrm{CV}$ was performed with a three-electrodepotentiostat [Bioanalytical Systems (BAS) 100B/W] in a grounded Faraday cage. A platinum-wire $(\mathrm{Pt})$ electrode was used as the counter electrode. A $\mathrm{Ag} / \mathrm{AgCl}(3 \mathrm{M}$ solution of $\mathrm{NaCl}$ ) electrode supplied by BAS was used as the reference electrode. ITO (size $4 \mathrm{~cm} \times 0.9 \mathrm{~cm}$ sheets, resistance $12 \Omega /$ sq.) electrode was used as the working electrode. All potentials were reported with respect to the $\mathrm{Ag} / \mathrm{AgCl}$ electrode at room temperature under an argon atmosphere. The FTIR spectra were recorded by a Varian 660-IR spectrometer. The Field Emission Scanning Electron Microscopy (FESEM) images of the modified electrode were obtained by a JSM-7500F field emission scanning electron microanalyzer (JEOL, Japan). A UV-vis spectrophotometer (NEOSYS-2000) purchased from SCINCO was used to conduct the spectroelectrochemical experiments to characterize the devices. The colorimetry measurements were performed using a Minolta CS-100 spectrophotometer.

Synthesis of copolymer

The potentiodynamic copolymerization of TT with EDOT was carried out by cycling the potential between +1.5 and $-1.5 \mathrm{~V}$ for $1-10$ cycles at $100 \mathrm{mV} / \mathrm{s}$ scan rate under an argon ambiance in $0.1 \mathrm{M} \mathrm{TBAP/MeCN}$. After electrolysis, the film was washed with $\mathrm{MeCN}$ to remove the excess supporting electrolyte and the unreacted monomers (Scheme 1). For the spectroelectrochemical studies, the copolymer was synthesized on an ITO-coated glass slide using a UV-cuvette as a single-compartment cell equipped with a $\mathrm{Pt}$ counter electrode and a $\mathrm{Ag} / \mathrm{AgCl}$ reference electrode. The electrochromic measurements, spectroelectrochemistry and switching studies of the copolymer film were studied after deposition on the ITO-coated glass slide, and were carried out in monomer-free 0.1 M TBAP/ $\mathrm{MeCN}$ solution systems. The surface area of ITO was $5 \times 2 \mathrm{~cm}^{2}$ and the copolymer was synthesized on approximately $3 \times 2 \mathrm{~cm}^{2}$ active area. The thickness of copolymer was measured about $23 \mathrm{~nm}$ for 10 cycles. The thickness was reduced when cycle numbers were less.

\section{Results and discussion}

Cyclic voltammetry

The CV experiments were carried out in the 0.1 M TBAP/ $\mathrm{MeCN}$ system on an ITO electrode at a scan rate $100 \mathrm{mV} / \mathrm{s}$. The redox behavior of EDOT was investigated via $\mathrm{CV}$, an electrochromism between deep blue and light blue colors 
Scheme 1 Electrochemical synthesis of P(TT-co-EDOT)

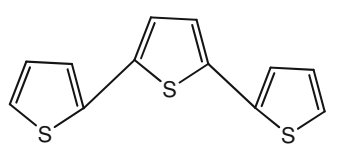

TT

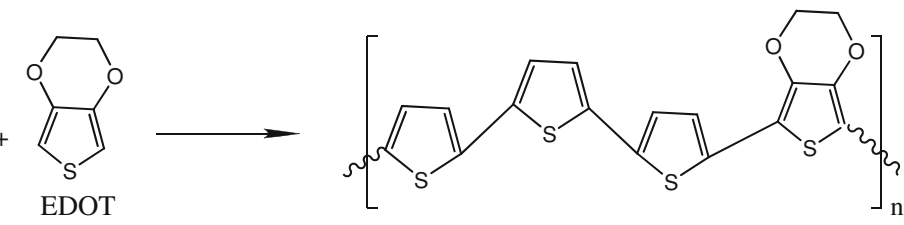

$\mathrm{P}$ (TT-co-EDOT) were observed. The CV of EDOT in $0.1 \mathrm{M}$ TBAP/MeCN showed that the oxidation of EDOT starts at $-0.46 \mathrm{~V}$ and reduction begins at $+1.13 \mathrm{~V}$. Reduction and oxidation peaks were found at -0.58 and $0.27 \mathrm{~V}$, respectively. In subsequent runs, the current increased with increasing scan number (Fig. 1a). From deep blue to light blue color was observed through total CV. The redox behavior of TT was investigated; the redox run was not as long as EDOT (Fig. 1b). A couple of reduction peaks were found at 0.56 and $-0.7 \mathrm{~V}$ and a pair of oxidation peaks were found at 1.43 and $-0.23 \mathrm{~V}$, respectively. An electrochromism between bluish purple and yellow orange colors was observed. In order to investigate the $\mathrm{CV}$ behavior of the copolymer, we performed CV for TT in the presence of EDOT (feed ratio $1 \mathrm{mM}: 1 \mathrm{mM}$ ) under the same experimental conditions. A radically changed voltammogram was recorded for P(TT-co-EDOT) copolymer. Redox current was increased between consecutive cycles and the reduction peak (at $0.4 \mathrm{~V}$ ) of the material being different from those of TT and EDOT, which indeed, could be interpreted as being due to the formation of the copolymer (Fig. 1c). When the redox behavior of the copolymer was investigated via $\mathrm{CV}$, an electrochromism between sky blue, gray, light purple, bluish violet and dark bluish violet colors were observed. The oxidation starts at $-0.25 \mathrm{~V}$ and reduction launches at $1.0 \mathrm{~V}$. P(TT-co-EDOT) was stable on the ITO electrode. For further electrochromic and others experiments 1:1 feed ratio was chosen.

\section{Infrared spectra}

The copolymer was characterized by FTIR. The FTIR spectra of PTT, PEDOT and P(TT-co-EDOT) are shown in Fig. 2. The curve a for PEDOT shows a strong peak at $1,091 \mathrm{~cm}^{-1}$ for $-\mathrm{C}-\mathrm{O}-\mathrm{C}-$ (in ring), a medium peak at $1,340 \mathrm{~cm}^{-1}$ for $\mathrm{C}-\mathrm{H}$ rocking, a strong peak at $1,519 \mathrm{~cm}^{-1}$ for $-\mathrm{C}-\mathrm{C}-$, a strong peak at $1,652 \mathrm{~cm}^{-1}$ for $-\mathrm{C}=\mathrm{C}-$, and a weak peak at $3,014 \mathrm{~cm}^{-1}$ for $=\mathrm{C}-\mathrm{H}$ (aromatic). In the spectrum of PTT curve $\mathrm{b}$, there are several peaks such as a strong and sharp peak at $790 \mathrm{~cm}^{-1}$ for $-\mathrm{C}-\mathrm{H}$, weak to string peak at $1,539 \mathrm{~cm}^{-1}$ for $-\mathrm{C}=\mathrm{C}-$ and $1,652 \mathrm{~cm}^{-1}$ for $-\mathrm{C}=\mathrm{C}-($ medium). Finally, the FTIR spectrum of P(TTco-EDOT) (curve c) had shown the characteristic peaks which were found in both PEDOT and PTT. For instance, $794 \mathrm{~cm}^{-1}$ was only found in PTT spectrum and
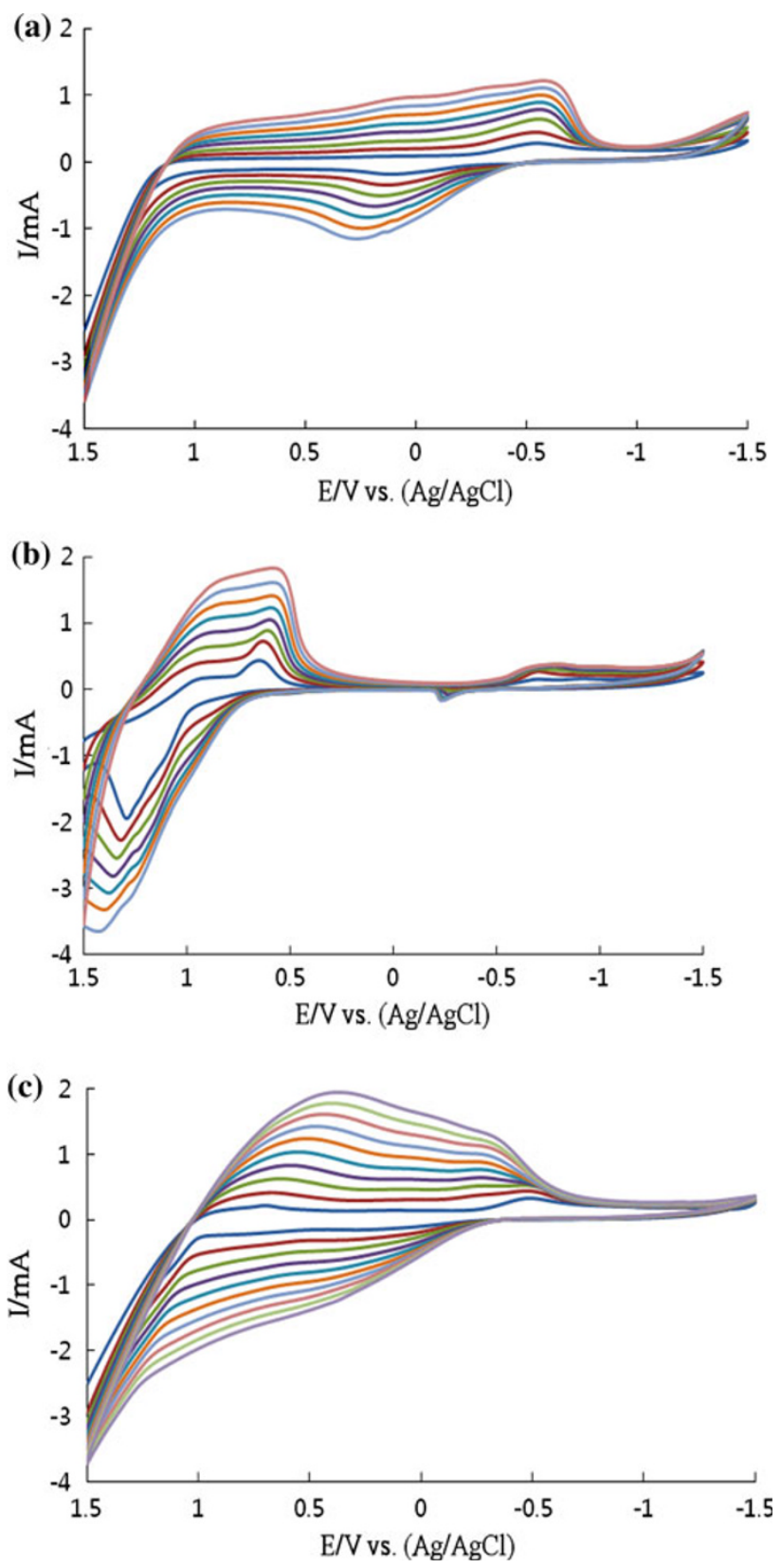

Fig. 1 CVs of a PEDOT, b PTT, c P(TT-co-EDOT) using ITO in $0.1 \mathrm{M} \mathrm{TBAP} / \mathrm{MeCN}$ at scan rate $100 \mathrm{mV} / \mathrm{s}$

$1,089 \mathrm{~cm}^{-1}$ and $1,344 \mathrm{~cm}^{-1}$ were only found in PEDOT spectrum. Nevertheless, other peaks were common for both PEDOT and PTT; for example, 1,521, 1,699 and 


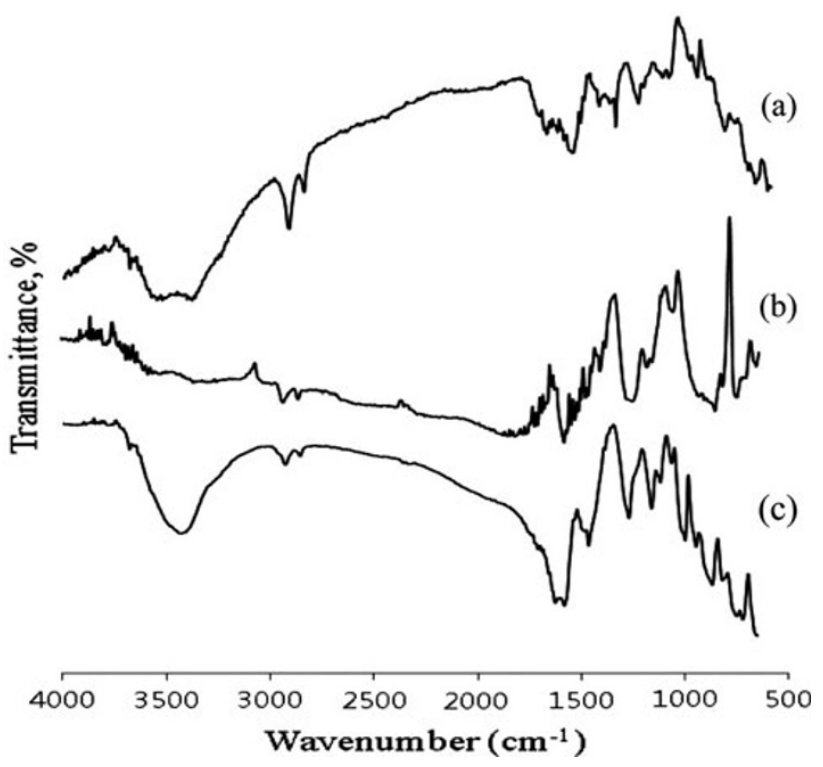

Fig. 2 FTIR spectra of a PEDOT, b PTT and $\mathbf{c}$ P(TT-co-EDOT)

$3,038 \mathrm{~cm}^{-1}$. All the above features indicated that P(TT-coEDOT) contains both TT and EDOT units.

\section{NMR spectroscopy}

${ }^{1} \mathrm{H}$ NMR spectrum for electrochemically synthesized copolymer was taken in $\mathrm{CDCl}_{3}$ (Fig. 3). Freshly prepared $5 \mathrm{mg}$ P(TT-co-EDOT) was taken into $4 \mathrm{ml} \mathrm{CDCl}_{3}$ and then ultrasonicated for $1 \mathrm{~h}$ to prepare a light blue colored solution (polymer attained sky blue color at $1.5 \mathrm{~V}$ ). $1 \mathrm{ml}$ of colored solution was taken for the NMR experiment after filtration. Chemical shifts $(\delta)$ were given relative to tetramethylsilane as standard. ${ }^{1} \mathrm{H}$ NMR $\left(300 \mathrm{Mz}, \mathrm{CDCl}_{3}\right) \delta_{\mathrm{H}}$ : $3.7(\mathrm{t}, 2 \mathrm{H}), 2.1(\mathrm{~s}, 1 \mathrm{H}), 1.8(\mathrm{~m}, 1 \mathrm{H})$, and $1.6(\mathrm{~d}, 1 \mathrm{H})$. Therefore, NMR data suggested that the copolymer film was synthesized successfully.

\section{Surface morphologies}

The surface morphologies of PTT, PEDOT and P(TT-coEDOT) were examined by SEM. The micrographs of the polymers show the excellence of their film formation ability. A wrinkled topography surface was observed from the PTT film (Fig. 4a) and the 3D net-like topography was observed on the PEDOT film (Fig. 4b). With regard to the copolymer of TT with EDOT (Fig. 4c), crystal-like topography was monitored on the P(TT-co-EDOT) film. The SEM micrograph showed a crystalline film that completely differed from PEDOT and PTT films. All films exhibit a homogeneous and compact structure. SEM experiment indicated that the copolymer formation was obtained from the electropolymerization in the presence of TT and EDOT on ITO-coated glass electrode.

\section{Spectroelectrochemistry of the copolymer}

The in situ electropolymerization occurred by cycling potential at $\pm 1.5 \mathrm{~V}$ for 10 cycles. Spectroelectrochemistry experiments provide key properties of conjugated polymers, such as band gap $\left(E_{\mathrm{g}}\right)$ and the intergap states that appear upon doping. The UV-vis spectra were taken at every 10-s interval. For the spectroelectrochemistry of the copolymer, the film was deposited on ITO via electrochemical polymerization; the P(TT-co-EDOT)-coated ITO was then investigated by UV-vis spectroscopy in monomer-free electrolytic system by switching the potential at $\pm 1.2 \mathrm{~V}$ (Fig. 5a). The intensity of the $\pi-\pi^{*}$ transition decreased while the applied potential was decreased. At the neutral state, $\lambda_{\max }$ value due to the transition of the copolymer was found to be $500 \mathrm{~nm}$ and $E_{\mathrm{g}}$ was calculated as $1.63 \mathrm{eV}$. Each scanning graph was separated and well organized and $\lambda_{\max }$ was increased gradually with decreasing potentials as can be seen in 3D graph in Fig. 5b.

Fig. $3{ }^{1} \mathrm{H}$ NMR spectrum of $\mathrm{P}$ (TT-co-EDOT) copolymer in $\mathrm{CDCl}_{3}$
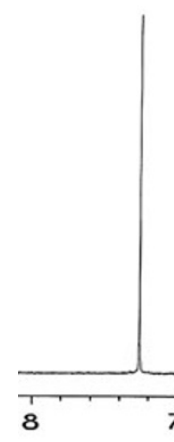

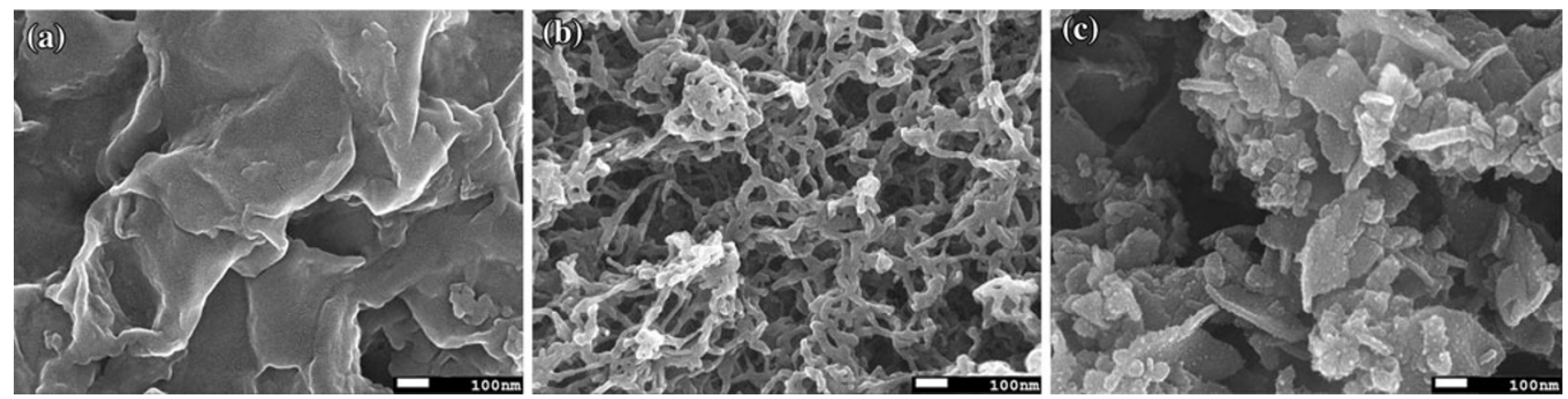

Fig. 4 SEM micrographs of a PTT, b PEDOT, $\mathbf{c}$ P(TT-co-EDOT) on ITO

Fig. 5 Spectroelectrochemical spectra of P(TT-co-EDOT) film on ITO in monomer-free $0.1 \mathrm{M}$ TBAP/MeCN at applied potential range of $\pm 1.2 \mathrm{~V}$ (a) and 3D diagram (b)

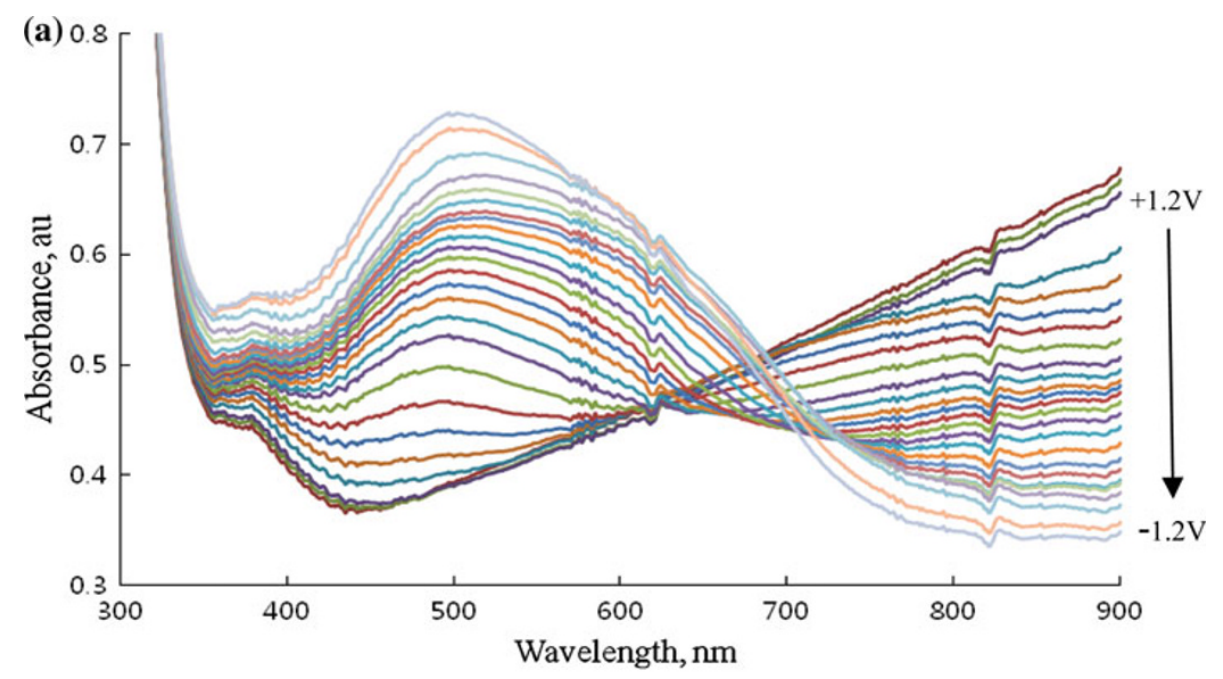

(b)

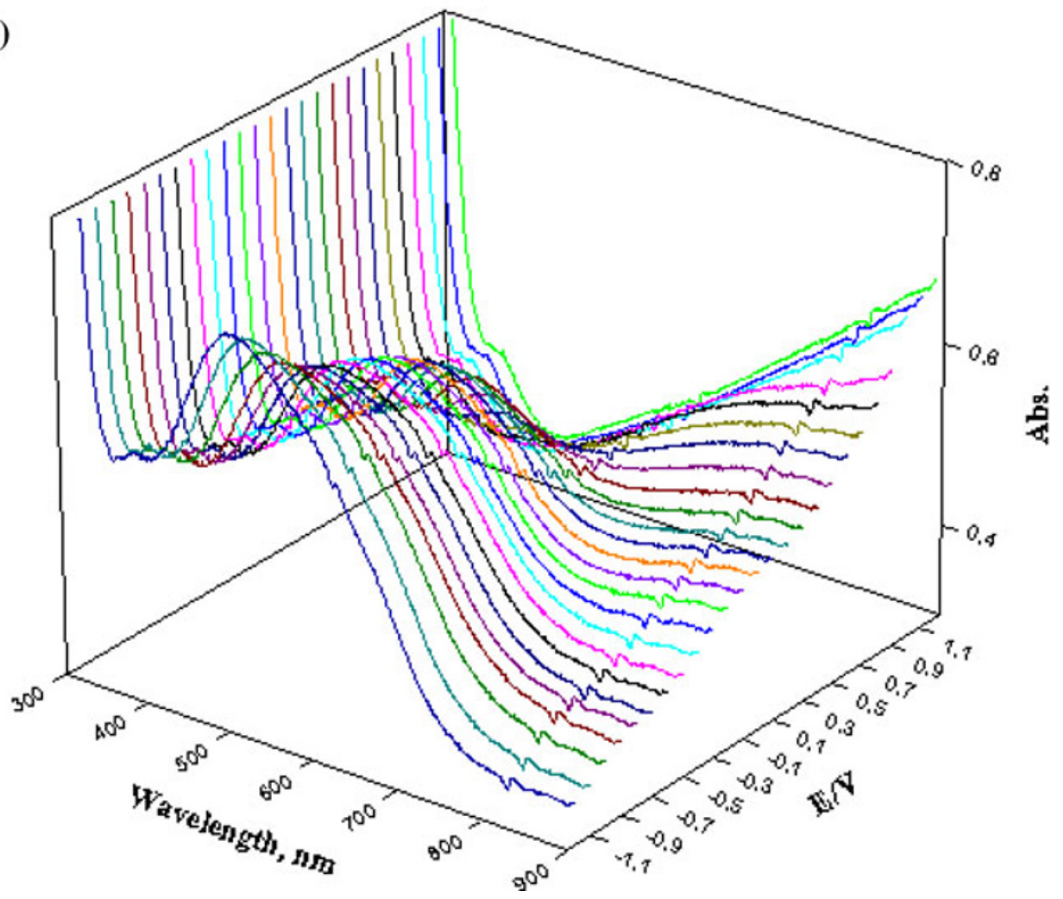


Scan rate dependence of the peak currents

The $\mathrm{CV}$ is the most widely used technique for acquiring qualitative information about electrochemical reactions. The current increases proportionally with the square root of the scan rate and concentration. These processes indicate that the negligible surface processes such as adsorption of reactants and products can occur and the reactants are soluble in solution (Skotheim and Reynoids 2007) and quasireversible and the redox process is not diffusion controlled, since the copolymer is immobilized at the electrode surface. Thus, investigation of peak current intensity with respect to scan rate indicates the nature of the electrochemical process being diffusion controlled and/ or the copolymer is well adhered to the electrode surface (Ahmed et al. 2011). The P(TT-co-EDOT) film was synthesized via cyclic potential electropolymerization $( \pm 1.5 \mathrm{~V})$ and the CVs of polymer film in the monomer-free solution were recorded at different scan rates (between 100 and $500 \mathrm{mV} / \mathrm{s}$ ) (Fig. 6). The polymer film coated on the working electrode was cycled between neutral and oxidized states at various scan rates to investigate the scan rate dependence in monomer-free TBAP/MeCN solution. The current response was directly proportional to the scan rate indicating that the copolymer film was electroactive and adhered well to the electrode. The scan rate for the anodic and cathodic peak currents showed a linear dependence as a function of the square root of scan rate as illustrated in Fig. 6 (inset). It was clear that the electroactive copolymer was well adhered to the working electrode surface.

Electrochromic properties of copolymer

Many methods have been used to identify and characterize color; the most widely used method to evaluate the color illuminated by a standard light source is the 1976 Commission Internationale de l'Eclairage (CIE) LAB (or $L^{*} a^{*}$ $b^{*}$ ) with the value of $L^{*}$ (luminance) representing how the

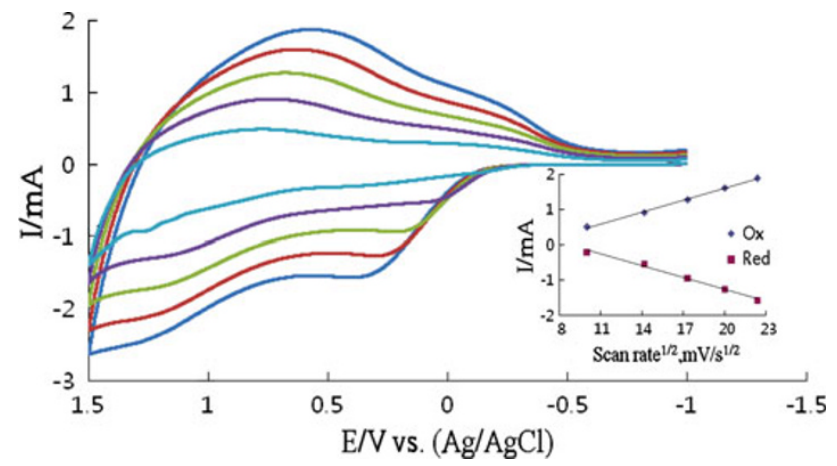

Fig. $6 \mathrm{CVs}$ for the P(TT-co-EDOT) film on ITO in monomer-free $0.1 \mathrm{M} \mathrm{TBAP} / \mathrm{MeCN}$ at different scan rates. Inset plot of peak current versus square root of scan rate measured material is light versus dark, the value $a^{*}$ (hue) representing how red versus green, and $b^{*}$ (saturation) representing how yellow versus blue the material is (Dyer et al. 2010). Three attributes of color; the $(a),(b)$ and $(L)$, were measured and recorded in Table 1 and can be seen in Fig. 7. The P(TT-co-EDOT) film has distinct electrochromic properties. It showed five different colors in the range from its neutral state to oxidized state upon various applied potentials.

\section{Electrochromic switching}

Response time, the time needed to perform switching between two states, is the most important characteristic of electrochromic devices. Another important parameter is the optical contrast, which can be defined as the transmittance difference between the redox states. Electrochromic switching studies were performed to test the ability of the polymer to switch rapidly and to exhibit a striking color change. The experiments carried out by spectroelectrochemistry showed the ability of P(TT-co-EDOT) to switch between its neutral and doped states with a resultant change in its transmittance at a fixed wavelength. During the experiment, the percentage transmittance $(\% T)$ of the polymer was measured using UV-vis spectrophotometer at $1,100 \mathrm{~nm}$. The polymer film was synthesized on ITOcoated glass slide and switched between +1.0 and $-0.5 \mathrm{~V}$ set period of time $5 \mathrm{~s}$. The contrast was measured as the $\% \Delta T$ difference between the reduced and oxidized forms of the copolymer and was found to be $33 \%$ at $550 \mathrm{~nm}$. As a matter of interest again, we found a great difference between the reduced and oxidized forms of the copolymer, $54 \%$ at $1,100 \mathrm{~nm}$. The insignificant decrease of $\% \Delta T$ was observed for higher thickness of the copolymer but the size does not interfered. Optical and current responses were monitored in switching experiments, and the responses were always uniform. These results confirm the good stability and reproducibility for electrochromic ability of P(TT-co-EDOT). As shown in Fig. 8, the stability and switching time $(<1 \mathrm{~s})$ of the copolymer were sensible. A comparative study is summarized in Table 2 .

Table 1 Electrochromic properties of the P(TT-co-EDOT) film

\begin{tabular}{lrllll}
\hline$\lambda_{\max }(\mathrm{nm})$ & $E / V$ & $L^{*}$ & $a^{*}$ & $b^{*}$ & Colors \\
\hline 500 & +1.5 & 56.43 & 0.69 & 8.70 & Sky blue \\
& +1.2 & 42.26 & 3.55 & 7.12 & Gray \\
& 0.0 & 30.02 & 4.32 & 4.70 & Light purple \\
-0.8 & 36.22 & 8.51 & 6.81 & Bluish violet \\
& -1.5 & 38.49 & 9.6 & 7.13 & Dark bluish violet \\
\hline
\end{tabular}




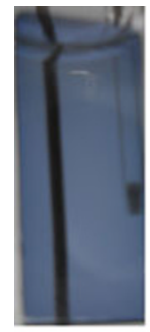

$1.5 \mathrm{~V}$

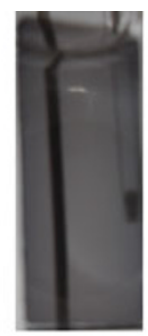

$1.2 \mathrm{~V}$

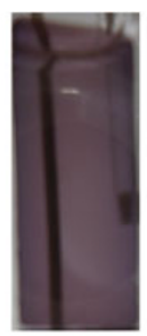

$0.0 \mathrm{~V}$

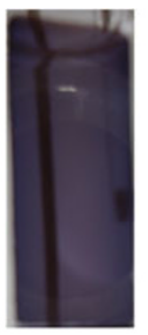

$-0.8 \mathrm{~V}$

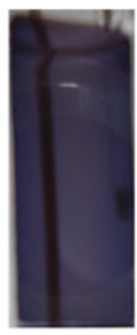

$-1.5 \mathrm{~V}$
Fig. 7 The colors are observed at different applied potentials for $\mathrm{P}(\mathrm{TT}-\mathrm{co}-\mathrm{EDOT})$ film; sky blue, gray, light purple, bluish violet and dark bluish violet, respectively
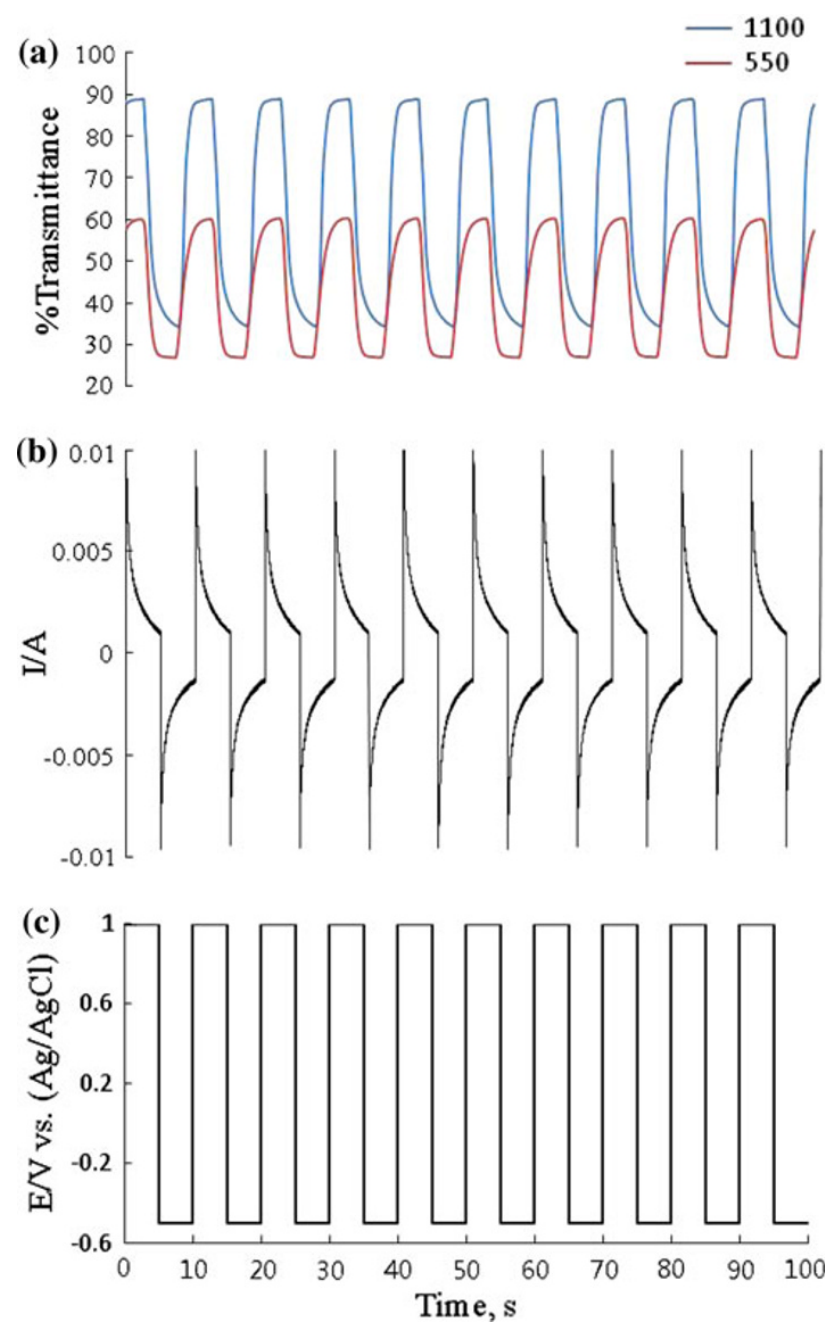

Fig. 8 Optical responses a at $550 \mathrm{~nm}$ and $1,100 \mathrm{~nm}, \mathbf{b}$ applied current and $\mathbf{c}$ applied voltage as a function of time for P(TT-coEDOT)

\section{Stability}

The stability of the devices toward multiple redox switching usually limits the utility of electrochromic materials in colored ECD applications. Hence, redox stability is an
Table 2 Comparison with other synthesized copolymers

\begin{tabular}{|c|c|c|c|c|c|}
\hline Name of polymer & $\begin{array}{l}E_{\mathrm{g}} \\
(\mathrm{eV})\end{array}$ & $\% \Delta T$ & $\lambda_{\max }$ & Colors & References \\
\hline P(DTTP-co-EDOT) & 1.65 & 18 & 470 & 4 & $\begin{array}{l}\text { Yigitsoy et al. } \\
\text { (2007) }\end{array}$ \\
\hline $\begin{array}{l}\mathrm{P}\left(\mathrm{SNS}-\mathrm{NH}_{2}-\mathrm{co}-\right. \\
\text { EDOT })\end{array}$ & 1.70 & 62 & 486 & 5 & $\begin{array}{l}\text { Yildiz et al. } \\
(2008)\end{array}$ \\
\hline P(MTNP-co-EDPT) & 1.71 & 30 & 500 & 5 & Ak et al. (2008) \\
\hline $\mathrm{P}(\mathrm{VC}-$-co-EDOT $)$ & 1.70 & 50 & 511 & 3 & $\begin{array}{l}\text { Ahmed et al. } \\
\text { (2011) }\end{array}$ \\
\hline P(TT-co-EDOP) & 1.65 & 31 & 750 & 3 & Seol et al. (2009) \\
\hline P(TT-co-EDOT) & 1.63 & 54 & 500 & 5 & Present work \\
\hline
\end{tabular}
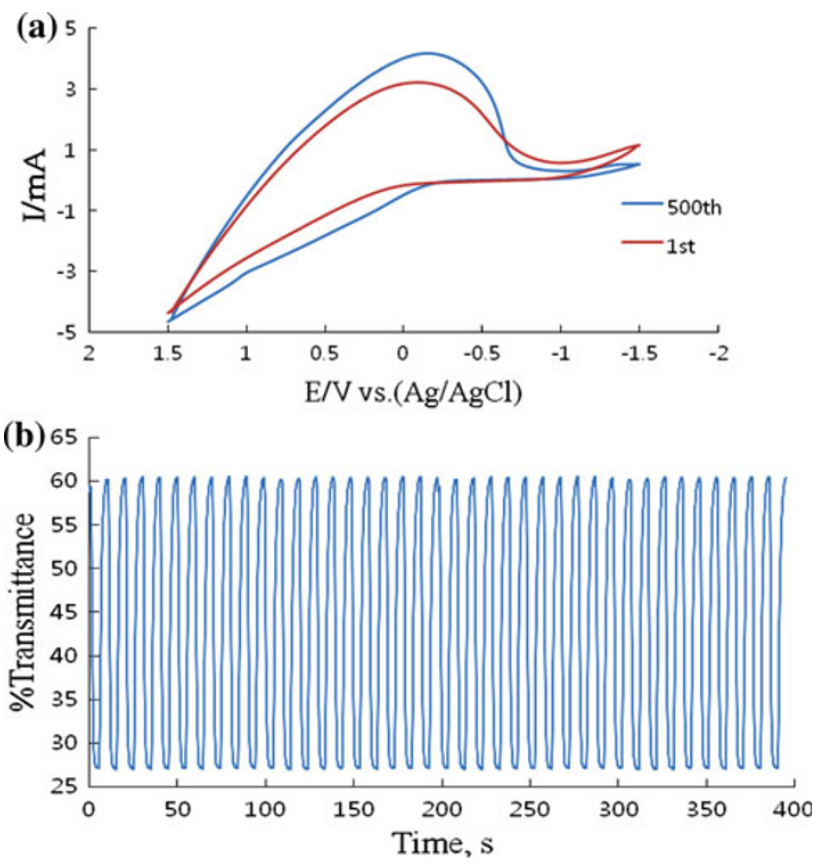

Fig. $9 \mathrm{CV}$ of $\mathrm{P}(\mathrm{TT}$-co-EDOT) for 1st cycle and 500th cycle at scan rate of $250 \mathrm{mV} / \mathrm{s}$ (a) and optical stability at $500 \mathrm{~nm}$ for long-time response (b)

important requirement for production of reliable electrochromic devices with long lifetimes. CV was employed to figure out the long-term stability of devices. Continuous cycling at $\pm 1.5 \mathrm{~V}$ for $\mathrm{P}$ (TT-co-EDOT) at scan rate of $250 \mathrm{mV} / \mathrm{s}$ was performed to analyze the stability of the device. The change in the anodic and cathodic peak currents as a function of the potential are presented in Fig. 9a. Device showed only a slight decrease in electroactivity accompanied by unperturbed color change from blue to dark bluish violet after 500 cycles. This result revealed that ECD has reasonable environmental and redox stability. To measure long-time switching stability, UV at $500 \mathrm{~nm}$ was applied (Fig. 9b). Switching square wave potentials were between $+1.0 \mathrm{~V}$ and $-0.5 \mathrm{~V}$ with a residence time of $5 \mathrm{~s}$. Optical contrast was decreased about $3 \%$ after $400 \mathrm{~s}$. 
Thermal analysis

Differential scanning calorimetry (DSC) was performed using DSC-60. DSC thermograms of PEDOT, PTT and $\mathrm{P}\left(\mathrm{TT}\right.$-co-EDOT) were examined in the range $0-280^{\circ} \mathrm{C}$ at a heating rate of $10^{\circ} \mathrm{C} \mathrm{min}{ }^{-1}$. DSC thermograms of PTT showed melting point $\left(T_{\mathrm{m}}\right)$ at $207.1^{\circ} \mathrm{C}$. DSC thermogram of $\mathrm{P}\left(\mathrm{TT}\right.$-co-EDOT) showed an endothermic process $T_{\mathrm{m}}$ at $224.7^{\circ} \mathrm{C}$. $T_{\mathrm{m}}$ was higher than (PEDOT has no $T_{\mathrm{m}}$ value) PTT due to cross-linking. DSC studies showed that thermal behavior of the copolymer was different from homopolymer film, which was synthesized under the same conditions.

\section{Conclusion}

In this study, a low band gap copolymer based on TT and EDOT was successfully synthesized via electrochemical polymerization. The polymerization of the P(TT-co-EDOT) copolymer was accomplished via $\mathrm{CV}$ method in the presence of TBAP $(0.1 \mathrm{M})$ in $\mathrm{MeCN}$ electrolyte at $\pm 1.5 \mathrm{~V}$. Characterization of the electrochemically synthesized polymer was carried out using CV, FTIR, SEM, spectroelectrochemical analysis, electrochromic switching and colorimetric studies. The band gap of the copolymer was found to be $1.63 \mathrm{eV}$. Moreover, electrochromic switching study results showed that the optical contrast $(\Delta T \%)$ was maximum, $54 \%$ at $1,100 \mathrm{~nm}$. According to the spectroelectrochemical analyses, the copolymer film showed excellent multicolor electrochromism (sky blue, gray, light purple, bluish violet and dark bluish violet). The copolymer of TT and EDOT can be a redox and optical stable device.

Acknowledgments This work was supported by a Special Research Foundation Grant funded by Chonnam National University (2011).

Open Access This article is distributed under the terms of the Creative Commons Attribution License which permits any use, distribution, and reproduction in any medium, provided the original author(s) and the source are credited.

\section{References}

Ahmed MS, Jeong H, You JM, Jeon S (2011) 9-Vinylcarbazole based semiconducting electrochromic copolymer with 3,4-ethylenedioxythiophene. Sci Adv Mater 3:289

Ak M, Tanyeli C, Akhmedov IM, Toppare L (2008) Optoelectrochemical properties of the copolymer of 2,5-di(4-methylthiophene-2-yl)-1-(4-nitrophenyl)-1H-pyrrole monomer with 3,4ethylenedioxythiophene. Thin Solid Films 516:4334

Albert KJ, Lewis NS, Schauer CL, Sotzing GA, Stitzel SE, Vaid TP, Walt DR (2000) Cross-reactive chemical sensor arrays. Chem Rev 100:2595

Bar G, Strum G, Gvishi R, Larina N, Lokshin V, Khodorkovsky V, Grinis L, Zaban A, Kiryuschev I (2009) A new approach for design of organic electrochromic devices with inter-digitated electrode structure. Solar Energy Mater Solar Cells 93:2118

Beaujuge PM, Ellinger S, Reynolds JR (2008) The donor-acceptor approach allows a black-to-transmissive switching polymeric electrochrome. Nat Mater 7:796

Dyer AL, Craig MR, Babiarz JE, Kiyak K, Reynolds JR (2010) Orange and red to transmissive electrochromic polymers based on electron-rich dioxythiophenes. Macromolecules 43:4460

Halls JJM, Walsh CA, Greenham NC, Marseglia EA, Friend RH, Moratti SC, Holmes AB (1995) Efficient photodiodes from interpenetrating polymer networks. Nature 376:498

Ho PK, Thomas DS, Friend RH, Tessler N (1999) All-polymer optoelectronic devices. Science 285:233

Kim H, Kim J, Kim H, Lee K, Kim J, Yoo D, Shin D (2010) A high efficiency photovoltaic module integrated converter with the asymmetrical half-bridge flyback converter. Sol Energy 84:1376

Lee DH, Liu YP, Lee KH, Chae H, Cho SM (2010) Effect of hole transporting materials in phosphorescent white polymer lightemitting diodes. Org Electron 11:427

Meng H, Tucker D, Chaffins S, Chen Y, Helgeson R, Dunn B, Wudl F (2003) An unusual electrochromic device based on a new lowbandgap conjugated polymer. Adv Mater 15:146

Monk PMS, Mortimer RJ, Rosseinsky DR (1995) Electrochromism: fundamentals and applications. VCH, Weinheim

Monk PMS, Mortimer RJ, Rosseinsky DR (2007) Electrochromism and electrochromic devices. Cambridge University Press, Cambridge

Mortimer RJ (1999) Organic electrochromic materials. Electrochim Acta 44:2971

Raimundo JM, Blanchard P, Frère P, Mercier N, Ledoux-Rak I, Hierle R, Roncali J (2001) Push-pull chromophores based on 2,2'-bi(3,4-ethylenedioxythiophene) (BEDOT) $\pi$-conjugating spacer. Tetrahedron Lett 42:1507

Ramirez CL, Parise AR (2009) Solvent resistant electrochromic polymer based on methylene-bridged arylamines. Org Electron 10:747-752

Roncali J (1997) Synthetic principles for bandgap control in linear $\pi$ conjugated systems. Chem Rev 97:173

Sahin E, Sahmetlioglu E, Akhmedov IM, Tanyeli C, Toppare L (2006) Synthesis and characterization of a new soluble conducting polymer and its electrochromic devices. Org Electron 7:351

Seol H, Jeong H, Jeon S (2009) Optoelectrochemical properties of copolymer of terthiophene with 3,4-ethlenedioxypyrrole. J Electroanal Chem 636:107-112

Skotheim TA, Reynoids JR (2007) Handbook of conducting polymers, 3rd edn. CRC press, Boca Raton

Sonmez G, Schwendeman I, Schottland P, Zong K, Reynolds JR (2003) N-substituted poly(3,4-propylenedioxypyrrole)s: high gap and low redox potential switching electroactive and electrochromic polymers. Macromolecules 36:639

Udum YA, Durmus A, Gunbas GE, Toppare L (2008) Both p- and n-type dopable polymer toward electrochromic applications. Org Electron 9:501

Wagner K, Pringle JM, Hall SB, Forsyth M, MacFarlene DR, Officer DL (2005) Investigation of the electropolymerisation of EDOT in ionic liquids. Synth Met 153:257

Yen HJ, Liou GS (2010) Novel blue and red electrochromic poly(azomethine ether)s based on electroactive triphenylamine moieties. Org Electron 11:299

Yigitsoy B, Varis S, Tanyeli C, Akhmedov IM, Toppare L (2007) Electrochromic properties of a novel low band gap conductive copolymer. Electrochim Acta 52:6561

Yildiz E, Camurlu P, Tanyeli C, Akhmedov I, Toppare L (2008) A soluble conducting polymer of 4-(2,5-di(thiophen-2-yl)-1H-pyrrol-1-yl)benzenamine and its multichromic copolymer with EDOT. J Electroanal Chem 612:247-256 
Yilmaz F, Cianga L, Guner Y, Toppare L, Yagci Y (2004) Synthesis and characterization of alternating copolymers of thiophene containing N-Phenyl maleimide and styrene via photo-induced radical polymerization and their use in electropolymerization. Polymer 45:5765
Yoo SJ, Cho JH, Lim JW, Park SH, Jang J, Sung YE (2010) High contrast ratio and fast switching polymeric electrochromic films based on water-dispersible polyaniline-poly(4-styrenesulfonate) nanoparticles. Electrochem Commun 12:164 